\title{
Utilização de localizadores eletrônicos foraminais na determinação da odontometria, em dentes decíduos
}

\author{
Electronic apex locators to root canal lenght determination, in primary teeth
}

\author{
Marília Pacífico LUCISANO \\ Aluna do Curso de Pós-Graduação em Odontopediatria - Departamento de Clínica Infantil, Odontologia Preventiva e \\ Social - Faculdade de Odontologia de Ribeirão Preto - USP - Universidade de São Paulo - Ribeirão Preto - SP - Brasil

\section{Mário Roberto LEONARDO} \\ Paulo NELSON-FILHO \\ Professor Titular - Departamento de Clínica Infantil, Odontologia Preventiva e Social - Faculdade de Odontologia de \\ Ribeirão Preto - USP - Universidade de São Paulo - Ribeirão Preto - SP - Brasil
}

\section{Raquel Assed Bezerra da SILVA}

Professora Doutora - Departamento de Clínica Infantil, Odontologia Preventiva e Social- Faculdade de Odontologia de Ribeirão Preto - USP - Universidade de São Paulo - Ribeirão Preto - SP - Brasil

\begin{abstract}
Resumo
A determinação do comprimento real de trabalho é uma etapa fundamental para o sucesso clínico, radiográfico e histológico do tratamento endodôntico, principalmente na dentição decídua, com o objetivo de minimizar injúrias periapicais e evitar alterações no germe dos dentes permanentes sucessores. O exame radiográfico é empregado na prática clínica para realização da odontometria, porém a determinação precisa do comprimento do canal radicular é dificultada devido a variações anatômicas, sobreposição de estruturas, distorções, erros técnicos ou erros na projeção. Os localizadores eletrônicos foraminais vêm sendo amplamente utilizados para a realização da odontometria em dentes permanentes, como precisão superior a 90\%. Nos últimos anos, esses aparelhos têm sido indicados, também, para dentes decíduos, reduzindo a exposição do paciente infantil à radiação e o tempo de trabalho clínico. O objetivo do presente trabalho será apresentar a indicação, vantagens e eficácia do método eletrônico para odontometria, bem como sua técnica de uso e resultados obtidos na odontometria de dentes decíduos.
\end{abstract}

\section{UNITERMOS}

Endodontia; dente decíduo; odontometria.

\section{INTRODUÇÃO}

A determinação do Comprimento Real de Trabalho é uma etapa de extrema importância durante a realização do tratamento endodôntico, particularmente na dentição decídua, a fim de minimizar possíveis injúrias à região periapical, reduzir a permanência de restos necróticos e bactérias no interior do canal radicular e evitar danos ao germe dos dentes permanentes sucessores ${ }^{2,3,8,19}$.
Sugere-se, portanto, atenção especial quando da determinação do comprimento de trabalho, uma vez que o sucesso clínico, radiográfico e histológico do tratamento está relacionado à adequada instrumentação e obturação do canal radicular, as quais devem se limitar ao canal dentinário. $\mathrm{O}$ canal dentinário corresponde à área histologicamente ocupada por tecido pulpar, restrita em seu extremo apical ao limite cemento-dentina-canal, onde inicia-se o canal cementário, que deve permanecer livre de qualquer 
intervenção. Assim, a constrição apical, e não o ápice radicular, constitui-se no ponto para o estabelecimento do limite apical de instrumentação, o qual identifica a profundidade que o preenchimento do canal, na obturação, poderá atingir ${ }^{22}$.

O exame radiográfico é tradicionalmente empregado na prática clínica para a determinação do comprimento de trabalho, bem como para obter informações a respeito da anatomia do canal radicular e dos tecidos periapicais ${ }^{11,14,20}$. Entretanto, a determinação precisa do comprimento do canal radicular é dificultada em função de variações anatômicas, da sobreposição de estruturas anatômicas, de erros técnicos ou erros na projeção, além de ser uma imagem bidimensional de uma estrutura tridimensional $1^{10,15,18,28}$. Em dentes decíduos, a presença dos germes dos dentes permanentes, em íntimo contato com os ápices dos dentes decíduos, muitas vezes também dificulta a observação dos limites apicais das raízes ${ }^{2,3}$.

Esses fatores têm estimulado o desenvolvimento de instrumentos eletrônicos para medir o comprimento do canal radicular, os quais podem localizar a posição da constrição apical com maior precisão.

O objetivo do presente trabalho será apresentar a indicação, as vantagens e a eficácia do método eletrônico para odontometria, bem como sua técnica de uso e resultados obtidos na odontometria de dentes decíduos.

\section{MÉtodo ELETRÔNICO PARA ODONTOMETRIA}

O método eletrônico tem sido estudado e aprimorado desde a metade do século passado, objetivando tornar mais precisa a determinação do comprimento real de trabalho ${ }^{22}$.

A primeira geração de localizadores eletrônicos foraminais, dispositivos tipo resistência, foi baseada na resistência elétrica existente entre a mucosa bucal e o ligamento periodontal ${ }^{12,14,30}$, enquanto que a segunda geração foi baseada no princípio da impedância. Entretanto, esses dispositivos apresentaram falhas, principalmente com relação à incapacidade de leitura em canais contendo soluções irrigadoras condutoras de corrente elétrica, restringindo seu uso ${ }^{22}$.

A terceira geração de localizadores eletrônicos foraminais, mais empregada atualmente, é representada por dispositivos do tipo impedância de dupla frequência, baseada em diferentes princípios elétricos ${ }^{4}$. Este princípio utilizado pelos localizadores recentes tem muitas vantagens quando comparado com os primeiros aparelhos lançados, especialmente porque são capazes de medir os canais radiculares em condições secas e úmidas, inclusive na presença de eletrólitos ${ }^{30}$.

A leitura da diferença de potencial elétrico dos tecidos propicia a execução de medidas eletrônicas. A parede dentinária do canal radicular exibe uma baixa condutibilidade elétrica, sendo que à medida que se aproxima do terço apical, a camada de tecido dentinário torna-se menos espessa, diminuindo sua capacidade de isolamento elétrico. Esta diminuição gradativa é interpretada eletricamente como uma diminuição da impedância da dentina. Os aparelhos baseados no método da frequência possuem uma calibragem tal que permite a indicação da variação de valores relativos de impedância (quociente ou diferença) da região apical, permitindo localizar a ponta do instrumento a aproximadamente $1 \mathrm{~mm}$ do forame apical, posição sugerida da constrição apical ${ }^{21}$. Os resultados do estudo de Oishi et al. ${ }^{25}$ (2002) que avaliaram a capacidade do aparelho Root ZX (Morita Corp. Tokyo. Japan) em detectar a presença de constrições apicais em dentes com ou sem patência foraminal, permitem-nos concluir que o princípio que rege o funcionamento dos localizadores eletrônicos foraminais do tipo frequência está vinculado ao fato das paredes do canal radicular possuírem uma impedância (capacidade que os materiais exibem de resistir à passagem de corrente elétrica) maior que o forame apical, representando, eletricamente, um modelo capacitor.

\section{EFICÁCIA dos localizadores ELETRÔNICOS FORAMINAIS}

Diversos estudos evidenciaram a eficácia dos localizadores eletrônicos foraminais na determinação da odontometria em dentes permanentes s, $^{5,9,30}$. Estudos comparando o método eletrônico com o radiográfico ${ }^{17,24}$ demonstraram diferentes resultados, devido ao fato dos aparelhos localizadores foraminais do tipo frequência indicarem a posição da constrição e saída foraminal, enquanto que a análise radiográfica interpreta apenas a posição do ápice radiográfico, coincidente em menos de $50 \%$ dos casos com a posição real do forame apical. Localizadores foraminais recentes como o Root ZX e o Bingo 1020 (Forum Engineering Technologies, Rishon Lezion, Israel), em estudo realizado por Kaufman et al. ${ }^{17}$ foram similares entre si e demonstraram serem mais precisos na mensuração do canal radicular quando comparados com o método radiográfico. Ainda neste estudo, várias soluções irrigadoras como o EDTA, a solução salina, a clorexidina e o hipoclorito de sódio foram testadas 
e nenhum destes produtos ocasionou alterações nas medidas obtidas.

A maior parte dos localizadores foraminais da geração atual não é afetada por soluções irrigadoras presentes no interior dos canais radiculares, sendo que o Root ZX tem sido o mais preciso na presença de hipoclorito de sódio ${ }^{12,13}$.

Os localizadores eletrônicos Apex Finder (Endo Analyser 8001; Analytic Technology, Redmond, WA, USA), Root ZX, Elements Diagnostic Unit Apex Locator (Sybron Endo, Sybron Dental, Anaheim, CA), RomiAPEX D-30 (Romidan LTD, KiryatOno, Israel), Mini Apex Locator (Sybron Endo, Sybron Dental, Anaheim, CA, USA), entre outros, são precisos na determinação da odontometria em dentes permanentes ${ }^{5,7,30}$ prevenindo a sobreinstrumentação ${ }^{7}$.

No entanto, é importante ressaltar que esses aparelhos devem ser associados a métodos radiográficos, com a finalidade de obter informações iniciais a respeito da anatomia do canal radicular ${ }^{22}$.

\section{USO DOS LOCALIZADORES ELETRÔNICOS FORAMINAIS EM DENTES DECÍDUOS}

A correta determinação do comprimento do canal radicular é uma das etapas mais importantes durante o tratamento endodôntico também em dentes decíduos ${ }^{6,19}$. Sem dúvida, o estabelecimento e a manutenção do limite apical de instrumentação são fatores críticos para o sucesso terapêutico, tornando a instrumentação mais segura e eficaz ${ }^{20}$.

Tratando-se particularmente da dentição decídua, tais procedimentos exigem maior cuidado e precisão, em função da presença dos germes dos dentes permanentes sucessores, em íntimo contato com seus ápices, e da ocorrência do processo de rizólise ${ }^{23,29}$.

Na dentição decídua, o método radiográfico tradicional apresenta limitações adicionais, incluindo a falta de exatidão ${ }^{6}$, particularmente nos casos onde a reabsorção fisiológica dos dentes decíduos ocorre nas superfícies vestibular ou lingual da raiz, as quais são de difícil visualização na radiografia ${ }^{20,23}$. Como consequência, há um aumento do risco de sobreinstrumentação e/ou sobreobturação, que pode reduzir de maneira significativa o sucesso clínico e histológico do tratamento, além de aumentar a possibilidade de danos aos germes dos permanentes ${ }^{23}$.

Em função do uso crescente dos localizadores eletrônicos foraminais para determinação do comprimento de trabalho e sua comprovada eficácia em dentes permanentes, nos últimos anos estes dispositivos vêm sendo indicados também para dentes decíduos.

Katz e colaboradores ${ }^{16}$, em 1996, relataram pela primeira vez a utilização do método eletrônico na dentição decídua, obtendo resultados precisos e confiáveis.

Algumas condições como diferentes tipos de eletrólitos, tamanho do forame e vitalidade pulpar podem influenciar na precisão dos localizadores apicais $^{27}$. Adicionalmente, fatores peculiares aos dentes decíduos como morfologia anatômica complexa, em particular dos molares, deposição de tecido mineralizado e presença de rizólise podem dificultar a determinação do comprimento real de trabalho ${ }^{16,19}$. Nestes dentes, a forma, dimensão e posição do forame apical são continuamente alteradas em função da reabsorção fisiológica ${ }^{19}$. No entanto, os localizadores eletrônicos foraminais podem superar essas restrições, sendo capazes de detectar os diâmetros mais estreitos do canal radicular ${ }^{27}$. Ainda, com a utilização deste método se reduz a exposição do paciente infantil à radiação e o tempo de trabalho, fatores de extrema importância na clínica odontopediátrica, além de não provocar dor e desconforto ao paciente, beneficiando o tratamento, principalmente no caso de crianças não colaboradoras ${ }^{16,19,27}$.

O método eletrônico tem se mostrado bastante preciso na determinação do comprimento de trabalho, quando comparado com medidas diretas do canal radicular, superando o método radiográfico, inclusive na presença de reabsorção fisiológica ${ }^{16,19,20,23}$.

O Root ZX é um dos localizadores eletrônicos foraminais que vem sendo mais utilizados nas pesquisas com dentes decíduos e tem mostrado resultados satisfatórios ${ }^{6,16,19,20,29}$. Este dispositivo foi avaliado in vivo em dentes decíduos, onde a determinação eletrônica do comprimento do canal radicular foi realizada clinicamente, e após a extração dos respectivos dentes, o comprimento "real" foi calculado usando as mesmas limas. As medidas obtidas por ambos os métodos foram comparadas e avaliadas segundo diferentes parâmetros clínicos, como tipo de dente e da raiz, vitalidade ou necrose pulpar e presença de reabsorção. Os resultados obtidos in vivo suportaram os resultados dos estudos in vitro, demonstrando a eficácia do Root $\mathrm{ZX}$, inclusive em casos de reabsorções radiculares pronunciadas ${ }^{19}$.

Os dispositivos Root ZX II (J Morita Corp., Tokyo, Japan), Mini Apex Locator, Endex (Osada, Tokyo, Japan) e Formatron D 10 (Parkell Electronic Division, NY, USA) também foram utilizados para determinar a 
confiabilidade do método eletrônico em dentes decíduos, evidenciando utilidade e exatidão $0^{6,20,23,27}$.

Além disso, a determinação eletrônica pode auxiliar outras medidas diagnósticas e aumentar a segurança do tratamento, principalmente em casos de lesões periapicais, canais estreitos e curvos e reabsorção fisiológica, situações que comumente ofecerem limitações ao exame radiográfico ${ }^{23,27}$.

Segundo Leonardo e colaboradores ${ }^{20}$ (2008), estes resultados favoráveis não implicam que as radiografias devam ser substituídas pelo uso dos localizadores foraminais. O método eletrônico deve ser considerado complementar ao método radiográfico de determinação do comprimento de trabalho, reduzindo o número de tomadas radiográficas. A avaliação radiográfica após a mensuração eletrônica confirma o trajeto do canal radicular, uma vez que a imagem da lima no interior do canal facilita a observação de detalhes anatômicos.

\section{PATOlogia PUlPar X PRECISÃo do MÉtodo ELETRÔNICO}

A influência da patologia pulpar na precisão e confiabilidade das medidas eletrônicas tem sido objeto de alguns estudos. Segundo Leonardo ${ }^{22}$ (2005) a condição da polpa, com vitalidade ou necrótica, não influencia a obtenção das medidas eletrônicas pelo método de impedância de frequências. No entanto, a presença de polpa inflamada no canal radicular dificulta a execução de mensurações eletrônicas, tendo em vista que há alteração do potencial de condutibilidade elétrica, uma vez que alterações patológicas neste tecido ocasionam mudanças em suas concentrações iônicas.

Akisue et al. ${ }^{1}$ (2007), com o objetivo de comparar a influência da condição pulpar (com vitalidade ou necrótica) na determinação do comprimento do canal radicular, usando um localizador eletrônico foraminal de quarta geração, verificaram que o dispositivo mostrou-se eficaz independentemente da condição pulpar.

Clinicamente, pode-se observar que as medições eletrônicas apresentam maior facilidade de aferição em canais com conteúdo pulpar necrótico, ou mesmo em casos de retratamentos. Portanto, recomenda-se que seja realizada uma pulpectomia parcial, seguida de abundante irrigação com solução de hipoclorito de sódio e posterior aspiração do excesso de solução irrigadora, para que se possa efetuar a mensuração sem interferências ${ }^{21}$.
Além disso, o instrumento utilizado para medição deverá ser compatível com o diâmetro anatômico do canal, uma vez que instrumentos mais calibrosos não alcançarão o terço apical, enquanto que instrumentos finos oferecem dificuldade de posicionamento apical e leitura, devido à falta de controle na penetração do instrumento ${ }^{22}$.

\section{SEQUÊNCIA OPERATÓRIA DE USO DE LOCALIZADORES ELETRÔNICOS FORAMINAIS ${ }^{21,22}$}

- Após a realização do isolamento absoluto do campo operatório e da abertura coronária, deve-se irrigar copiosamente o canal radicular com solução de hipoclorito de sódio, atentando-se para o fato que a câmara pulpar deve permanecer seca, ou seja, a substância irrigadora não pode exceder as entradas dos canais;

- Inicialmente deve-se ligar o aparelho e instalar os eletrodos no intermediário da lima e na comissura labial do paciente;

- Anteriormente à colocação da lima no interior do canal radicular, é necessário que se realize a patência do canal, com uma lima manual de diâmetro compatível com o mesmo, no comprimento de trabalho provisório, determinado a partir da radiografia para diagnóstico;

- Neste momento, acopla-se o porta-lima à lima, que deverá, então, ser inserida no interior do canal radicular, certificando-se que a mesma se ajuste às paredes internas. Neste momento, executa-se a leitura;

- Ao aproximar-se do comprimento real de trabalho, um alarme sonoro intermitente será acionado. Deve-se então continuar com a lima no sentido apical até o alarme tornar-se contínuo. Neste momento, a marcação refere-se à posição da saída foraminal e deve-se, então, recuar a lima até o ponto relativo à posição da constrição apical;

- Atingido o comprimento real de trabalho, deslizase o cursor até o ponto de referência escolhido.

A execução de exame radiográfico após odontometria eletrônica é um procedimento muito controver$\mathrm{so}^{26}$. No entanto, cabe salientar que a finalidade básica da tomada radiográfica não é confirmar a precisão do limite apical estabelecido pela leitura eletrônica, mas sim cumprir a função de reconhecimento do trajeto do canal e de acrescentar detalhes adicionais à radiografia inicial $^{2,3}$. 


\section{Conclusão}

Os localizadores eletrônicos foraminais foram introduzidos recentemente para o tratamento endodôntico de dentes decíduos. No entanto, estudos têm avaliado esses aparelhos na determinação da odontometria nesses dentes, independentemente da presença do processo de reabsorção fisiológica, evidenciando sua eficácia, inclusive em nível clínico.

Estudos adicionais são necessários a fim de embasar o emprego clínico em dentes decíduos dos diferentes localizadores eletrônicos foraminais disponíveis comercialmente.

\begin{abstract}
Working length determination is an extremely important step of the endodontic treatment. It is particularly critical in primary teeth in order to minimize periapical injury and possible damage to the permanent successor tooth germ. Radiography is traditionally used for root canal length determination. However, an accurate working length determination is dependent on anatomical variations, interference of anatomical structures or errors in projections. Electronic apex locators have been used for working length determination in permanent teeth with up to $90 \%$ accuracy. Over the last years, these devices have also been indicated for primary teeth, reducing the exposure of children to x-rays and the clinical chairtime. The purpose of the present study was to discuss the indications, advantages and the efficacy of electronic working length determination. Additionally, this paper describes a clinical sequence and the published results after using these devices in primary teeth.
\end{abstract}

\title{
UNITERMS
}

Endodontics; primary tooth; odontometry.

\section{REFERÊNCIAS}

1. Akisue E, Gavini G, Figueiredo JAP. Influence of pulp vitality on length determination by using the elements diagnostic unit and apex locator. Oral Sur Oral Med Oral Pathol Oral Radiol Endod. 2007;104:129-32.

2. Assed S. Tratado de Odontopediatría. Tomo 2. Caracas: Amolca; 2008.

3. Assed S. Odontopediatria: bases científicas para a prática clínica. São Paulo: Artes Médicas; 2005.

4. Baldi JV, Victorino FR, Bernardes RA, Moraes IG, Bramante CM, Garcia RB, et al. Influence of embedding media on the assessment of electronic apex locators. J Endod. 2007;33:476-9.

5. Bernardes RA, Duarte MAH, Vasconcelos BC, Moraes IG, Bernardineli N, Garcia RB, et al. Evaluation of precision of length determination with 3 electronic apex locators: Root ZX, Elements Diagnostic Unit and Apex Locator, and RomiAPEX D-30. Oral Sur Oral Med Oral Pathol Oral Radiol Endod. 2007;104:91-4.

6. Bodur H, Odabas M, Tulunoglu O, Tinaz AC. Accuracy of two different apex locators in primary teeth with and without root resorption. Clin Oral Investig. 2007;12:137-41.

7. D' Assunção FLC, Albuquerque DS, Salazar-Silva JR, Ferreira LCQ, Bezerra PM. The accuracy of root canal measurements using the Mini Apex Locator and Root ZX-II: an evaluation in vitro. Oral Sur Oral Med Oral Pathol Oral Radiol Endod. 2007;104:50-3.

8. Dandashi MB, Nazif MM, Zullo T, Elliott MA, Schneider LG, Czonstkowsky M. An in vitro comparison of three endodontic techniques for primary incisors. Pediatr Dent. 1999;15:254-6.

9. Dotto RF, Renner D, Barletta FB, Dotto SR, Wagner MH. Evaluation about precision of the electronic apex locator NOVAPEX in working length measurement. Rev Odontol da Univ Cidade de São Paulo. 2005;3:263-9.
10. El Ayouti A, Weiger R, Lost C. The ability of root ZX apex locator to reduce the frequency of overestimated radiographic working length. J Endod. 2002;28:116-9.

11. Forsberg J. Radiographic reproduction of endodontic "working length" comparing the paralleling and the bisecting-angle techniques. Oral Sur Oral Med Oral Pathol. 1987;64:353-60.

12. Gordon MPJ, Chandler NP. Electronic apex locators. Int Endod J. 2004;37:425-37.

13. Grimberg F, Banegas G, Chiacchio L, Zmener O. In vivo determination of root canal length: a preliminary report using the Tri Auto ZX apex-locating handpiece. Int Endod J. 2002;35:590-3.

14. Haffner C, Folwaczny M, Galler K, Hickel R. Accuracy of electronic apex locators in comparison to actual length- an in vivo study. J Dent. 2005;33:619-25.

15. Hoer D, Attin T. The accuracy of electronic working length determination. Int Endod J. 2004;37:125-31.

16. Katz A, Mass E, Kaufman AY. Electronic apex locator: A useful tool for root canal treatment in the primary dentition. J Dent Child. 1996;63:414-7.

17. Kaufman AY, Keila S, Yoshpe M. Accuracy of a new apex locator: an in vitro study. Int Endod J. 2002;35:186-92.

18. Keller ME, Brown CE Jr, Newton CW. A clinical evaluation of the Endocater - an electronic apex locator. J Endod. 1991;17:271-4.

19. Kielbassa AM, Muller U, Munz I, Monting JS. Clinical evaluation of the measuring accuracy of ROOT ZX in primary teeth. Oral Sur Oral Med Oral Pathol Oral Radiol Endod. 2003;95:94-100.

20. Leonardo MR, Silva LA, Nelson-Filho P, Silva RA, Raffaini MS Ex vivo evaluation of the accuracy of two electronic apex locators during root canal length determination in primary teeth. Int Endod J. 2008;41:317-21. 
21. Leonardo MR. Comprimento real de trabalho fundamentos e técnicas (localizadores eletrônicos foraminais). In: Ramos CAS, Bramante CM. Endodontia. Tratamento de canais radiculares- princípios técnicos e biológicos. São Paulo: Artes Médicas; 2005. v.1,p.945-76.

22. Leonardo MR. Endodontia. Tratamento de canais radiculares-princípios técnicos e biológicos. São Paulo: Artes Médicas; 2005.v.1.

23. Mente J, Seidel J, Buchalla W, Koch MJ. Electronic determination of root canal length in primary teeth with and without root resorption. Int Endod J. 2002;35:447-52.

24. Muthu S, Rajendran N, Rajan M, Sundaresan B. Evaluation of working length determination methods: An in vivo/ ex vivo study. Ind J Dent Res. 2007;18:60-2.

25. Oishi A, Yoshioka T, Kobayashi C, Suda H. Electronic detedtion of root canal constrictions. J Endod. 2002;28:361-4.

26. Reche MEA, Ramos CAS. Influência da determinação eletrônica do comprimento de trabalho, comprovada ou não radiograficamente, na qualidade do nível de obturação dos canais radiculares. Estudo "in vivo". [monografia] .Londrina: Universidade Norte do Paraná; 2001.

27. Subramanian P, Konde S, Mandanna DK. An in vitro comparison of root canal measurement in primary teeth. J Ind Soc Pedod Prev Dent. 2005;23:124-5.
28. Surmont P, D'Hauwers R, Martens L. Determination of tooth length in Endodontics. Rev Belge Med Dent. 1992;47:30-8.

29. Tosun G, Erdemir AU, Eldeniz U, Sermet U, Sener Y. Accuracy of two electronic apex locators in primary teeth with and without apical resorption: a laboratory study. Int Endod J. 2008;41:436-41.

30. Venturi M, Breschi L. A comparison between two electronic apex locators: an ex vivo investigation. Int Endod J. 2007;40:362-73.

Recebido em 15/09/08 Aprovado em 29/07/09

Correspondência: Prof. Dr. Paulo Nelson-Filho Departamento de Clínica Infantil, Odontologia Preventiva e Social Faculdade de Odontologia de Ribeirão Preto Universidade de São Paulo Av. do Café, s/n - Monte Alegre Ribeirão Preto - SP - Brasil CEP: $14040-904$ e-mail: nelson@forp.usp.br 